\title{
Health-Related Quality of Life in MS: Issues and Interventions
}

Can. J. Neurol. Sci. 2009; 36: 540-541

Multiple sclerosis (MS) is one of the leading causes of neurological disability among young adults and has potentially enormous implications for health-related quality of life (HRQL) from diagnosis onward. This issue of the Canadian Journal of Neurological Sciences includes two papers which address HRQL among MS patients: Hopman et $\mathrm{al}^{1}$ examine physical and mental HRQL over time, while Abou Zeid et $\mathrm{al}^{2}$ focus on gait apraxia which could negatively impact HRQL. Both papers further our understanding of factors and interventions which may influence HRQL. They also illustrate some of the complexities of HRQL research which may influence study results and complicate their interpretation.

Hopman et $\mathrm{al}^{1}$ observed that MS patients' physical and mental HRQL summary scores on the Medical Outcomes Study Short Form-36 fell well below Canadian normative data at baseline. Both summary scores were relatively stable over a twoyear follow-up period, but there was a significant decline in the physical function subscale of the physical summary score and a significant improvement in the social function subscale of the mental summary score. This is consistent with previous research which indicates that physically-oriented dimensions of HRQL tend to decline while mentally-oriented dimensions remain stable or even improve over time, possibly due to patient psychosocial adjustment or shift in values ${ }^{3,4}$. It also points out that the way in which HRQL is measured, in this case summary scores versus subscale scores, may influence the results observed. In addition, length of follow-up and attention to statistical versus clinical significance may have a bearing on interpretation. For example, Hopman et $\mathrm{al}^{1}$ point out that, while the decline in physical function was statistically significant, at least four years of follow-up would be needed to reach clinical significance at the small rate of change observed.

Moderate to severe disability, progressive disease course, use of a variety of health services and interferon therapy were among the variables which were negatively associated with patients' physical HRQL summary score at baseline in Hopman et al's ${ }^{1}$ multivariate analysis. The authors interpreted this to mean that patients with poorer function and lower HRQL were more likely to be using services and taking interferon. However they also noted that the impact of interferon therapy on change in physical HRQL over time was negligible. Likewise disability, progressive disease course, service use and interferon therapy were negatively associated with patients' mental HRQL summary score at baseline, but the impact of interferon therapy over time was negligible. Abou Zeid et $\mathrm{al}^{2}$ concluded that gait apraxia can cause considerable functional impairment in MS patients, but they also noted that $47 \%$ of their study participants were on MS therapies with no observed advantage. Since the Abou Zeid et $\mathrm{al}^{2}$ study was cross-sectional, and although longitudinal the Hopman et $\mathrm{al}^{1}$ study did not report any HRQL measure taken before interferon therapy, it was not possible to assess the initial impact of MS therapies on gait apraxia or HRQL.

Hopman et $\mathrm{al}^{1}$ also observed that unemployment and lower education were negatively associated with both MS patients' physical and mental HRQL at baseline in multivariate analyses. This observation suggests the importance of assessing the influence of socio-demographic factors on HRQL, with employment status and education level being possible candidates for intervention. While MS patients who are not working are probably unable to do so as a consequence of their disease, it is likewise possible that those with higher education have access to employment opportunities which are physically less demanding; more flexible in terms of work location, hours and retraining; and more appealing in terms of income, medical, health, disability and /or retirement benefits. As the authors remark, longitudinal studies are needed to assess the nature of the association between employment status, education level and HRQL in MS patients, which have also been observed in other cross-sectional studies 5 .

Very few factors were associated with change in HRQL over time in the multivariate analyses of Hopman et al's ${ }^{1}$ data. While the use of most health services measured was not associated with change over time, the two factors associated with a better outcome were forms of social/health service support. There was a positive association between living with someone or having a caregiver and slower rate of decline/improvement in physical HRQL summary score, and a positive association between the use of visiting nurse services and slower rate of decline/ improvement in mental HRQL summary score. Although the former association was only a trend rather than statistically significant, small effects over time on HRQL may be common in chronic diseases such as MS and justify attention to this and other trends reported by Hopman et $\mathrm{al}^{1}$. Both associations confirm the role of support (formal or informal, personal or professional, individual or group) in HRQL found by other researchers ${ }^{6}$ and reinforce the importance of investigating sociodemographic factors and non-pharmacological interventions that might influence HRQL. Abou Zeid et $\mathrm{al}^{2}$, noting that pharmacological treatment for gait apraxia is not wellestablished, also mention the possible value of other types of intervention including physical therapy retraining and the provision of adequate assistive devices to improve function and safety in patients with gait disorders.

Since the majority of persons with MS are diagnosed around age 30 and their lifespan is often not shortened by more than five to ten years ${ }^{7}$, many will live with a reduced and possibly declining HRQL for decades. Finding ways to maintain, or better yet, improve HRQL is a significant concern for both researchers and clinicians involved in caring for MS patients. Despite the challenges inherent in HRQL research, including recognition of 
the influence which HRQL measures may have on the assessment of outcomes and the need for longitudinal studies, more research is required to understand HRQL in MS. In particular, since neither Hopman et $\mathrm{al}^{1}$ nor Abou Zeid et $\mathrm{al}^{2}$ among other researchers ${ }^{8,9}$ have observed a clear association between disease modifying therapies and HRQL or improvements in physical or mental HRQL over time during treatment, non-pharmacological interventions should be explored. More attention might also be paid to appropriate timing of interventions since Hopman et al's ${ }^{1}$ results, like The Canadian Burden of Illness Study ${ }^{10}$, suggest that there may be a substantial initial decline in HRQL followed by some stabilization. A better understanding of the factors associated with HRQL may assist in developing targeted interventions which can be tested for their effectiveness in improving HRQL.

Sharon Warren

Karen V.L. Turpin

Kenneth G. Warren

Edmonton, Alberta

\section{REFERENCES}

1. Hopman WM, Coo H, Pavlov A, Day A, Edgar CM, McBride EV, et al. Multiple sclerosis: change in health-related quality of life over two years. Can J Neurol Sci. 2009;36:554-61 .

2. Zeid NEA, Weinshenker BG, Keegan BM. Gait apraxia in multiple sclerosis. Can J Neurol Sci. 36:562-5.

3. Solari A, Ferrari G, Radice D. A longitudinal survey of self-assessed health trends in a community cohort of people with multiple sclerosis and their significant others. J Neurol Sci. 2006;243 (12):13-20.

4. Singer MA, Hopman WM, MacKenzie TA. Psychological adjustment in four chronic medical conditions. Qual Life Res. 1999;8(8):687-91.

5. Warren S, Turpin KVL, Pohar SL, Jones CA, Warren KG. Comorbidity and health-related quality of life in people with multiple sclerosis. Int J MS Care. 2009;11:14-20.

6. McCabe MP, Stokes M, McDonald E. Changes in quality of life and coping with multiple sclerosis over a 2 year period. Psychol Health Med. 2009;14(1):86-96.

7. Warren S, Warren KG. Multiple sclerosis. World Health Organization, Geneva, Switzerland; 2001.

8. Zivadinove R, Zorzon M, Tommasi MA, Nasulli D, Bernardi M, Monti-Bragadin L, et al. A longitudinal study of quality of life in patients with multiple sclerosis treated with interferon beta-1a. J Neurol Sci. 2003;216:113-8.

9. Simone IL, Ceccarelli A, Tortorella C, Bellacosa A, Pellegrini F, Plasmati I, et al. Influence of interferon beta treatment on quality of life in multiple sclerosis patients. Health Qual Life Outcomes. 2006;4:96-103.

10. The Canadian Burden of Illness Study Group. Burden of illness in multiple sclerosis. II. Quality of life. Can J Neurol Sci. 1998;25: $31-8$. 